\title{
Glioblastoma Multiforme of Optic Nerve in Adults with Hyper intense Diffuse Weighted Imaging
}

\author{
Karthik Adiraju ;Phani Harshitha Yarlagadda;Sohil Patel \\ (General Medicine,Kamineni Institute Of Medical Sciences,India ) \\ (General Medicine,Kamineni Institute Of Medical Sciences,India) \\ (Fellow NeuroRadiology,Massachusettes General Hospital,Boston,U.S)
}

\begin{abstract}
We present a case of glioblastomamultiforme of the optic nerve with Hyper intense Diffusion Weighted Imaging $(D W I)$ in a 56 year old man without Neurofibromatosisl who presented with generalized tonic clonic seizures and loss of vision in right eye. Presence of hyperintenseDWI imaging of optic nerve makes this case unique.Further details about diagnostic methods available with major emphasis on Magnetic Resonance Imaging in adults is been done.
\end{abstract}

Keywords: GlioblastomaMultiforme,Gliomas,Imaging options of GBM,Optic Nerve Tumors,Optic nerve thickening.

\section{Introduction:}

Glioblastoma Multiforme of the optic nerve a rare kind of cancer, usually slow-growing and found in children. It is rarely found in individuals over the age of 20. It has also been associated with the genetic disorder neurofibromatosis Type 1(NF1). Generally optic nerve gliomas are not hyperintense on diffusion weighted imaging(DWI) .It is mostly seen in conditions of infarction and papilledema.The malignant optic glioma of adulthood, on the other hand, is an extremely rare optic pathway tumor. Till 1990, only 30 cases were reported in this century. In a review of literature in 2004 by Bettina Wabbles et al, 45 cases of adult malignant optic gliomas have been described. Of these patients $51 \%$ were male, $49 \%$ females; the mean age at diagnosis was 54 years (median $59 \mathrm{yrs}$, range 22 to $79 \mathrm{yrs}$ ). The most common sign of ONG is progressive loss of vision and may lead to death in children.

\section{Casepresentation}

A 56 years old man presented with complaints of loss of vision in the right eye since 2days. Itwas not associated with any pain or discharge. There was also a history of irritability and generalized tonic clonic seizures since two to three years. His general physical examination was normal. Best corrected visual acuity in the left eye was 6/24 .Anteriorsegment examination revealed sluggish pupillary reaction in both the eyes. On fundus examination, no papilloedemaor any vessel occlusion was not seen bilaterally. The color vision on Ishiara's chart was 5/14 in the left eye and none in the left eye. On confrontation test, temporal fields of both right and left eyes were decreased.

With this background, an MRI of the brain was done on 1.5 Tesla Siemens Essenzasystem. We found that there was thickening of the right optic nerve (Fig1)showinghyperintense signal on T2W/ FLAIR images(Fig2). The lesion appeared hypointense to isointense on T1W images and showed hyper intensity on DWI. The right optic nerve appear expanded. The intracanalicular optic nerves appeared normal with thickening of intraorbital part on right side(Fig3). The post contrast images (using $20 \mathrm{ml}$ of Gadodiamin revealed a small (size) enhancing focus in posterior part of optic nerve on the right side. Rest of the lesion showed no significant enhancement.

The patient was then taken up forbiopsy.Histopathological examination of the biopsy showed cerebral tissue with varying degree of cellularity, characteristic cyto-morphology, abundant mitotic figures, increased vascularity consistent with a diagnosis of glioblastomamultiforme.(Fig4)

\section{Discussion}

Gliomas account for $40-50 \%$ of all primary and metastaticintracranial tumors. Glioblastoma is the most common typeof glioma.WHO 2000 classification grades astrocytic tumors fromgrade I to grade IV. WHO Grade I tumor includes pilocyticastrocytoma, pleomorphic xanthoastrocytoma andsubependymal giant cell astrocytoma. Other astrocytomasinclude diffuse astrocytoma (grade II), anaplasticastrocytoma(grade III) and glioblastoma (grade IV).

Gliomas of the optic pathway are classified as (a) the relatively benign optic nerve glioma (typically occurring in the pediatric age group) and (b) the malignant optic glioma of adulthood .Benign optic nerve gliomas represent $4 \%$ of orbital tumors, $4 \%$ of intracranial gliomas, and $2 \%$ of intracranial tumors. They also 
constitute two thirds of all primary optic nerve tumors. The benign optic nerve glioma is more common and is considered a low grade Astrocytoma. From 10 to $38 \%$ of pediatric patients with optic nerve glioma have NF-1; conversely, $15-40 \%$ of children with NF-1 have opticnerveglioma. Bilateral optic nerve gliomas are almost pathognomonic for NF-1.

The malignant optic glioma of adulthood, on the other hand, is an extremely rare optic pathway tumor. Till 1990, only 30 cases were reported in this century. In a review of literature in 2004 by Bettina Wabbles et al, 45 cases of adult malignant optic gliomas have been described. Of these patients $51 \%$ were male, $49 \%$ females; the mean age at diagnosis was 54 years (median 59yrs, range 22 to $79 \mathrm{yrs}$ )

The sites of occurrence of malignant optic glioma in addition to the optic chiasma and the optic nerve(s) were found to be the hypothalamus in $50 \%$ of patients, the temporal lobe in $22.5 \%$ and the basal ganglia in $15 \%$. Other more infrequent sites were the midbrain, parietal cortex, cerebellum and the cervical, thoracic and lumbar subarachnoidalspaces. The malignant optic glioma of adulthood is classified pathologically as either ananaplastic astrocytoma or a glioblastomamultiforme . In adults, malignant optic gliomas are rare and are rapidly fatal visual pathway tumors. Anatomical classification forOPGs was proposed by Dodge et al in 1958, defining tumors asinvolving either the optic nerves alone (Stage 1), the chiasm with or without nerveinvolvement (Stage 2), and the hypothalamus or other adjacent structures (Stage 3). The modified Dodge classification further categories stage 1 into stage 1a (i.e. involvement of Single optic nerve), 1b(Bilateral Optic nerve involvement), 1c (Cisternal segment of the optic nerve). Stage 2 is categorized into $2 \mathrm{a}$ (Central chiasmatic) and $2 \mathrm{~b}$ (Asymmetric chiasmatic).Stage $3 \mathrm{a}$ is optic tracts involvement, $3 \mathrm{~b}$ is asymmetric tract involvement. Stage 4 is diffuse posterior tract involvement and stage $4 \mathrm{~b}$ is asymmetric posterior tract involvement. The Hypothalamic involvement, leptomeningeal dissemination and associated neurofibromatosis are also taken in account (i.e. $\mathrm{H}+/-, \mathrm{LM}+/-$ and $\mathrm{NF} 1+/-$ )

\section{Signs And Symptoms Of Optic Nerve Glioma}

The most common sign of ONG is progressive loss of vision .Other includes: Changes in visual acuity,proptosis may occur in later stages, involuntary eye movements, changes in appetite and sleep, neurologic deficits, nausea, headache and vomiting. Rarely, childhood Optic Nerve Glioma can result in death.Symptoms of an optic nerve glioma are caused by the tumor pressing against the nerves

\section{Imaging Optic Nerve Gliomas:}

5.1RADIOGRAPHY films used orbits imaged ANGIOGRAPHY not currently used examines blood vessels around the orbit. COMPUTED TOMOGRAPHY great soft tissue definition, also visualizes bony detail.

5.2MAGNETIC RESONANCE IMAGING is currently GOLD STANDARD in tumor imaging Superior contrast resolution of soft tissue structures.Gliomas generally demonstrate high signal intensity on $\mathrm{T} 2 \mathrm{w}$ images and low signal intensity on $\mathrm{T} 1 \mathrm{w}$ images, unless hemorrhage or calcification is present. Low grade astrocytoma (WHO grade I and II) tend to be well defined, nonhemorrhagic and demonstrate little mass effect, vasogenic edema or heterogeneity. Anaplastic astrocytomas (WHO grade III) are less well defined and demonstrate moderate amount of mass effect, heterogeneity and edema with varying degrees of enhancement. Glioblastoma (WHO grade IV) are poorly defined and often have considerable mass effect, vasogenic edema, hemorrhage and heterogeneity. Irregular ring enhancement with nodularity and necrotic foci are typical of Glioblastoma .

5.3DIFFUSION WEIGHTED IMAGING (DWI)Apart from conventional and contrast MR, glioma grading have been further attempted on the basis of DWI.It is a form of MR imaging based upon the diffusion of water molecules within a voxel. The greater the cellularity, the greater the diffusion restriction, e.g. tumors.DWI can be used for early identification of ischemic stroke,differentiation of epidermoid cyst from arachnoid cyst ,abscess,cortical lesions in CJD ,differentiation of herpes encephalitis from diffuse temporal gliomas, extent of diffuse axonal injury, active MS plaque (old plaques will not be bright) The signal intensity of gliomas on DWI is variable (hyper-, iso-, or hypointense) but no case in literature has been reported with hyper intense DWI in GBM of optic nerve.DWI and/or ADC maps may not be reliable to distinguish neoplastic cell infiltration from peritumoral edema in patients with malignant glioma .

5.4MAGNETIC RESONANCE SPECTROSCOPY (MRS) MRI with non-invasive characterization of tissue spectroscopic patterns of gliomas different than normal brain tissue potentially help in delineating tumour spread VISUAL EVOKED POTENTIALS (VEPs) uses electroencephalography to evaluate conduction of visual pathway from optic nerve through optic chiasm to occipital cortex useful in detecting anterior visual pathway disturbance Findings indicate that results may not be reliable for children under 5 years old (peak age for ONG).MRS shows increased Cho values in glioma with associated decrease in NAA/Cho and $\mathrm{Cr} / \mathrm{Cho}$ ratios with lipid-lactate peaks. One study has shown that specific metabolites, when standardized to water, are of diagnostic value in the division of tumors into three categories. Specifically, the lactate/water ratio can be used 
to differentiate GBMs, anaplastic astrocytomas, and low-grade tumors. The choline/water, choline/creatine, and lactate/creatine ratios can be used to distinguish high-grade from low-grade tumors (5).

5.5MR PERFUSION STUDIESshows a correlation between relative cerebral blood volume and tumor grade.

5.6PET-CT SCAN Helpful in staging of the disease Effectively locates the tumour which is useful for treatment Can assess tumour progression/recession following treatment Allows the possibility for a less invasive technique that could easily replace more invasive ones PET-MRI SCAN Same application as a PET-CT scanner Better soft tissue than CT More expensive.

\section{Figures:}

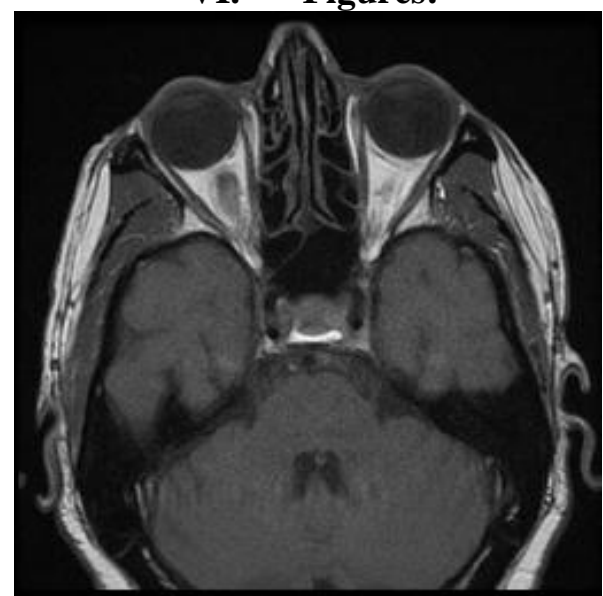

Fig 1.optic nerve thickness on right side

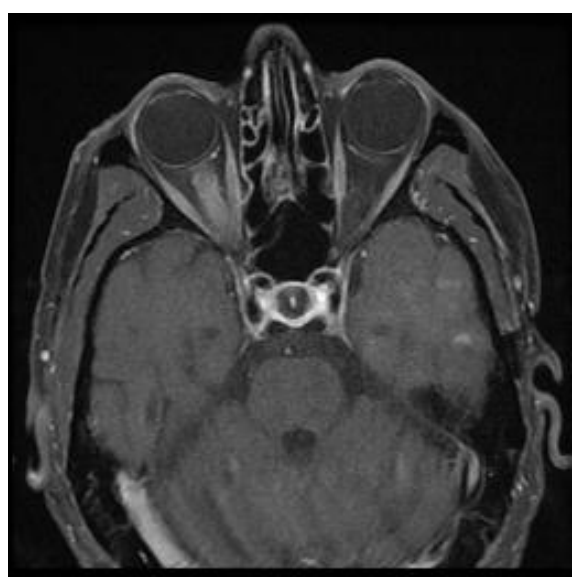

Fig2.Hyperintense imaging of Optic Nerve

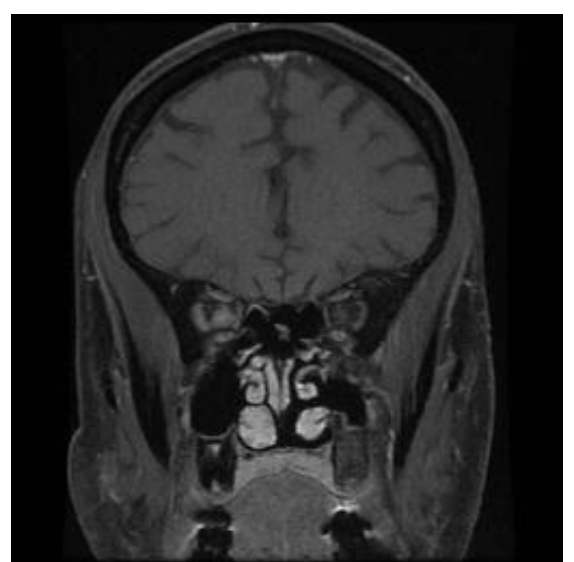

Fig3.Intraorbital optic nerve thickening 


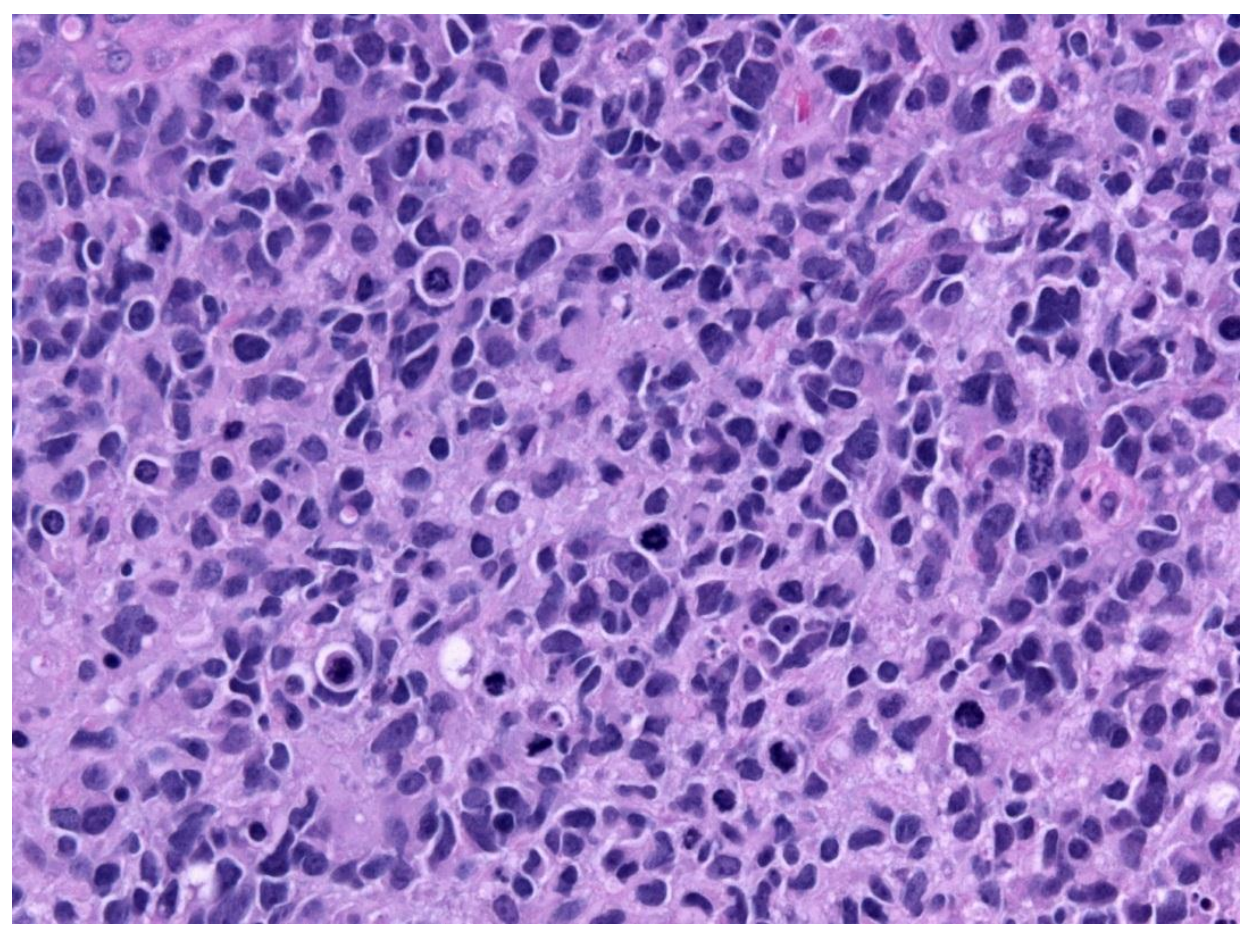

Fig 4.sMitotic Activity of GBM specimen

\section{Treatment Options}

To halt the progression in vision loss or tumour growth .Types of treatment include: surgery, chemotherapy and radiation therapy .CHEMOTHERAPY has now been determined as the best form of treatment for all ages. If Chemotherapy fails, radiation therapy is then used.RADIATION THERAPY: Major side effects of cranial irradiation: Mental retardation Endocrinopathies Cerebrovascular disease Fortunately side effects are less common today due to treatments that are more precisely focused on Abnormal tissue .This patient is on chemotherapy and under follow up.

\section{Conclusion}

Glioblastoma are very rare in adults compared to children.They are generally malignant in adults resulting in loss off vision. GBM of optic nerve can present with hyperintense DWI . The possibility of tumor cannot be excluded in differential diagnosis of hyper intense DWI in optic nerve apart from infarction and edema. New imaging and treatment options can be used for earlier detection, more accurate treatment planning and better prognosis.

\section{References}

[1]. Agamanoliz, D.P. (n.d.). Neuropathology. http://www.neuropathologyweb.org/ .

[2]. Auntminnie Case of the Day. (2008) Childhood Optic Nerve Glioma.

[3]. Carlson, A.H. (2007). Siemens Demonstrates Prototype MRI-PET System.

[4]. Gujar, S., Maheshwari, S., Bjorkman-Burtscher, I. \&Sundgren, P. (2005) Magnetic resonance spectroscopy. Journal of Neuroopthalmology, 25 (3), 217-226.

[5]. Hollander, M.D., Fitzpatrick, M., O’Connor, S.G., Flanders, A.E., Tartaglino, L.M. (1999). Optic Gliomas.Optic pathway glioma: correlation of imaging findings with the presence of neurofibromatosis. American Journal of Neuroradiology, 22, 1963-1969.

[6]. Listernick, R., Louis, D., Packer, R. \&Gutmann, D. (1997) Optic Pathway Gliomas in Children with Neurofibromatosis 1: Consensus Statement from the NF1 Optic Pathway Glioma Task Force. Annals of Neurology , 41 (2), 143-149. Liu, G.T. (2006). Optic gliomas of the anterior visual pathway.

[7]. Dean BL, Drayer BP, Bird CR, et al. Gliomas: Classification with MR Imaging. Radiology 174: 411-415.

[8]. Richards J Hicks: Supratentorial adult brain tumors. In Clinical MRI Robert Edelman et al (third ed.) Philadelphia, PA. Saunders Elsevier, 2006 pp 1098-1100.

[9]. Sugahara T, Korogi Y, Kochi M, et al. Usefulness of diffusion-weighted MRI with echo-planar technique in the evaluation of cellularity in gliomas. J MagnResonImaging .

[10]. Taylor T, Jaspan T, Milano G, Gregson R, Parker T, Ritzmann T, Benson C, Walker D. Radiological classification of optic pathway gliomas: experience of a modified functional classification system. The British Journal of Radiology, 2008.

[11]. Woiciechowsky C, Vogel S, Meyer R, Lehmann R. Magnetic resonance imaging of a glioblastoma of theoptic chiasm. J Neurosurg 83:923-925.

[12]. Taphoorn MJB, de Vries-Knoppert WAEJ, PonssenH,Wolbers JG. Malignant optic glioma in adults. J Neurosurg 1989;70:277-279.

[13]. Bilaniuk LT, Atlas SW, Zimmerman RA. The orbit.In: Lee SH, Rao KCVG, Zimmerman RA, eds. Clinical MRI and CT. New York, NY: McGraw-Hill; 1992:119-19 